\title{
On the Colloidal Stability of Spherical Copolymeric Micelles
}

\author{
Álvaro González García, ${ }^{\dagger, \ddagger, \S}$ Alessandro Ianiro, ${ }^{\ddagger, \S}$ and Remco Tuinier*, ${ }^{*}, \ddagger$ (i) \\ ${ }^{\dagger}$ Van 't Hoff Laboratory for Physical and Colloid Chemistry, Department of Chemistry \& Debye Institute, Utrecht University, \\ Padualaan 8, $3584 \mathrm{CH}$ Utrecht, The Netherlands \\ ${ }^{*}$ Laboratory of Physical Chemistry, Department of Chemical Engineering and Chemistry, \& Institute for Complex Molecular \\ Systems (ICMS), Eindhoven University of Technology, P.O. Box 513, 5600 MB Eindhoven, The Netherlands
}

Supporting Information

ABSTRACT: Using self-consistent field (SCF) calculations, we systematically quantify the pair interactions between spherical diblock copolymer micelles following a bottom-up approach. From the equilibrium properties of self-assembling micelles at different separation distances, a simple yet insightful pair interaction can be extracted. The SCF results match with an analytical model based upon closed expressions for the free energy change per diblock copolymer in the micelle. To gain insights into the colloidal stability of dilute micelle suspensions, the second virial coefficient normalized by the undistorted micelle volume $\left(B_{2}^{*}\right)$ is evaluated. For stable

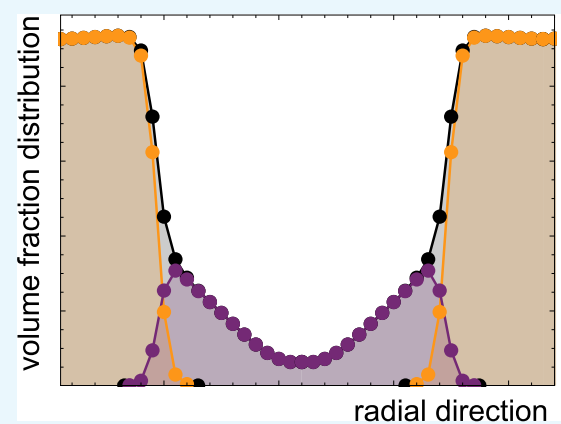

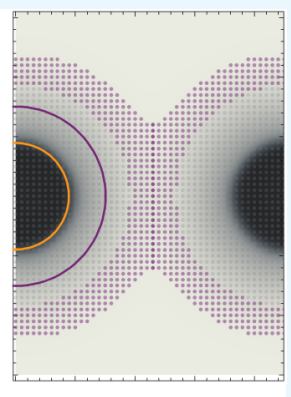
micelles $\left(B_{2}^{*} \gtrsim-6\right)$, we find a weak dependence of $B_{2}^{*}$ on solvophilic block length for varying core-forming block properties (core solvation and block length). The micelle suspension gets unstable $\left(B_{2}^{*} \lesssim-6\right)$ when the corona-forming block crosses $\Theta$-solvent conditions toward poor solvency. In contrast with what is expected from models where the soft nature of the micelle is not taken into account, increasing the effective grafting density of solvophilic tails from the core then leads to colloidal destabilization of the micelle suspension.

\section{INTRODUCTION}

The association of polymers and surfactants (macromolecules) into soft colloidal particles provides a playground for generating a wide range of self-assembled architectures in selective solvents. ${ }^{1}$ Substantial attention has been paid to predetermine the preferred morphology of association colloids. $^{2}$ Among the possible micellar shapes, the spherical one is appealing because of its wide applicability for instance in coatings, ${ }^{3}$ in food, ${ }^{4}$ and as drug delivery system. ${ }^{5-8}$ In many applications, control is not only desired over the morphology of the self-assembled structure but also over the thermodynamic stability of the micellar suspension. A widely applied technique to enhance the stability of inorganic colloidal particles is grafting polymers onto their surface, which leads to steric stabilization. ${ }^{9,10}$ For spherical micelles formed by block copolymers, such steric stabilization is inherent. ${ }^{11}$ Understanding how micelles interact is key to envisage, and therefore predict, the stability of a micellar suspension. Previously presented models for micelle-micelle interactions account for the core as a hard surface onto which the solvophilic components are tethered. ${ }^{12-14}$ Micelles are however dynamic because the assembled molecules are in equilibrium with free ones, so the core-corona interface is soft and dynamic. ${ }^{15-18}$ For micelles with large coronal domains, the interaction between micelles mediated by overlap of coronas has been compared with that of star-like polymers. ${ }^{19,20}$ It is noted, however, that star-like polymers are not selfassembled structures, ${ }^{21}$ and such models hence neglect the presence of free diblock copolymer in solution. In this paper, the interaction between diblock copolymer spherical micelles is quantified, while accounting for their soft polymeric and associative nature. When computing the micelle-micelle interaction potential, we allow the aggregation number (the number of polymers composing the micelle) to equilibrate with free polymer in the bulk at each intermicelle separation ( $r$ ) distance. We account for intermicellar distances $r \geq 2 R_{\mathrm{h}}^{\mathrm{o}}$, with $R_{\mathrm{h}}^{\mathrm{o}}$ the hydrodynamic radius of an undistorted micelle. Hence, we focus on dilute micelle suspensions rather than on high-density solid phases of micelles ${ }^{19}$ or possible micelle morphology transformations above their overlap concentration. 22,23

An effective indicator for the thermodynamic stability of a colloidal suspension is the second osmotic virial coefficient $B_{2}{ }^{24,25}$ Experimentally measured $B_{2}$ values for micelle suspensions are limited. ${ }^{26-30}$ The value of $B_{2}$ can be used to specify the (colloidal) stability of a suspension. For a collection of hard spheres, $B_{2}^{*}=B_{2} / v_{c}=4,{ }^{31}$ where $v_{c}$ is the volume of the colloidal particle considered. If repulsive forces beyond the pure hard core excluded volume interaction are present between the colloidal particles, $B_{2}^{*}>4$. For monocomponent systems of interacting spheres, the Vliegenthart-Lekkerkerker

Received: September 27, 2018

Accepted: December 7, 2018

Published: December 21, 2018 
criterium $^{32}$ identifies the onset of colloidal gas-liquid coexistence at $B_{2}^{*}=B_{2} / v_{\mathrm{c}} \lesssim-6^{33,34}$

Here, we present a bottom-up approach to study the stability of a (colloidal) suspension of spherical micelles formed by diblock copolymers. We calculate the pair interaction potentials via self-consistent field (SCF) calculations for block copolymers with different block length and solubility. Results are compared with an alternative analytical expression for the interaction potential based upon the thermodynamics of micelle formation. ${ }^{35}$ Numerical SCF computations and analytical results are in good agreement. Furthermore, we calculate the normalized second virial coefficient, $B_{2}^{*}=B_{2} / v_{\mathcal{O}}$ where $v_{c}$ is the volume of an isolated micelle, and evaluate its dependency on block copolymer composition and solvency parameters of the blocks.

\section{RESULTS AND DISCUSSION}

First, we evaluate the dependence of the equilibrium micelle properties on the intermicelle distance. This yields pair potentials obtained via different methods (explained in the Methods section), for which case-examples are presented. Subsequently, we use these pair potentials to compute the second virial coefficients mediated by the solvophilic block solubility and chain length. Finally, the effect of the diblock copolymer composition and monomeric interaction parameters on the colloidal stability is summarized into two comprehensible, simple plots.

Equilibrium Properties of Micelles with Varying Intermicelle Distance. We focus first on the changes of the micellar equilibrium properties at different intermicelle separation distances $(x)$. These micellar properties were studied using a lattice with concentration gradients in one (spherical lattice) or two directions (cylindrical lattice). In Figure 1 , we present the grand potential $(\Omega)$ obtained via

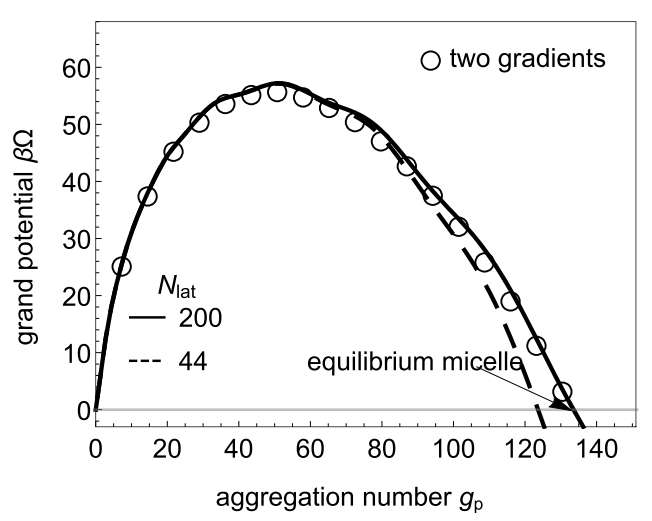

Figure 1. Grand potential $\Omega$ as a function of the aggregation number $g_{\mathrm{p}}$ at two different intermicelle distances $r$ obtained using a spherical lattice (solid and dashed curves). Open circles correspond to the grand-potential curve considering a single micelle in a cylindrical lattice, allowing to study spherical micelles with concentration gradients in two directions. The micelle considered is composed of $\mathrm{B}_{24} \mathrm{~A}_{45}$ diblock copolymers with $\chi_{\mathrm{BW}}=2, \chi_{\mathrm{AW}}=0.4$, and $\chi_{\mathrm{AB}}=1$.

Scheutjens-Fleer self-consistent, mean-field (SCF) computations as a function of the aggregation number $g_{\mathrm{p}}$ for different lattice sizes and types for micelles formed by diblock copolymers $\mathrm{B}_{24} \mathrm{~A}_{45}$ in a solvent $\mathrm{W}$. The interaction between blocks and of blocks with the solvent are specified via FloryHuggins interaction parameters, namely $\chi_{\mathrm{BW}}=2$ and $\chi_{\mathrm{AW}}=0.4$.
The interaction parameter $\chi_{\mathrm{AB}}=1$ is used in all our calculations. The grand-potential curves using one or two directions for concentration gradients practically overlap if the lattice dimensions are large enough; in such a case, the dilute solution limit of individual micelles is reached. There are no appreciable differences in the maximum free energy required to form a micelle as micelles get closer (decreasing $x$ ). However, the average equilibrium aggregation number of the micelle (which satisfies $\Omega=0$ with $\partial \Omega / \partial g_{p}<0$ ) decreases when micelles are formed at small enough distances. For all diblock sequences $\mathrm{B}_{m} \mathrm{~A}_{n}$ studied here, it was verified that the preferred self-assembled structure is a spherical micelle. An analysis of the preferred self-assembled morphology of similar block sequences has been investigated via SCF computations and compared with experimental results. ${ }^{36}$

The corresponding equilibrium concentration profiles are presented Figure 2. If the number of lattice sites is sufficiently large, the micelle size and aggregation numbers are independent of the lattice type considered (spherical or cylindrical lattice). This can be appreciated by the projection of the equilibrium sizes from the spherical lattice onto the cylindrical one (left panel of the top row in Figure 2). All sizes are expressed in terms of lattice units (l.u., see Methods). Note that the distance between the centers of the micelles $x$ is set by the number of lattice sites. For one-gradient SCF computations, $x=2 N_{\text {lat }}$ (with $N_{\text {lat }}$ the number of concentration shells considered). For two-gradient computations, $x=2 N_{\text {lat }}^{\mathrm{r}}$ (if the nearest micelles are in the radial direction) or $x=N_{\text {lat }}^{\text {y }}$ (in case the nearest micelles are in the longitudinal direction). Further details are deferred to the Methods section. The hydrodynamic micelle radius $\left(R_{\mathrm{h}}^{\mathrm{o}}\right)$ in the dilute limit $\left(x \gg 2 R_{\mathrm{h}}^{\mathrm{o}}\right)$ is $R_{\mathrm{h}}^{\mathrm{o}} \approx 20$ l.u. for the $\mathrm{B}_{24} \mathrm{~A}_{45}$ block copolymer micelle (details on the calculation of $R_{\mathrm{h}}^{\mathrm{o}}$ deferred to the Supporting Information). From the concentration profiles, the hydrodynamic size can be computed. The solvophobic blocks are concentrated in the core of the spherical micelle, which is compact and nearly solvent-free. ${ }^{37}$ The approximated core size $\left(R_{c}\right)$ is indicated via the orange dashed vertical lines. The solvophilic blocks are mainly located in the corona, which is well-solvated. For this case, the hydrodynamic size (vertical purple dashed lines) matches with the situation at which the total polymer volume fraction has roughly decayed to $10 \%$. It is noted that solvophilic polymer segments are also significantly present at positions beyond $R_{\mathrm{h}}^{\mathrm{o}}$, see top panels of Figure 2 . We denote the region where solvophilic segments are clearly present $(\phi \gg$ $\phi_{\text {bulk }}$, with $\phi_{\text {bulk }}$ the polymer bulk concentration) beyond $R_{\mathrm{h}}^{\mathrm{o}}$ as the solvophilic tails part. When micelles get close, the overlapping of these outer tail regions leads to a contraction of the coronas already at intermicelle distances $x>2 R_{\mathrm{h}}^{\mathrm{o}}$. This induces a decrease in the micelle size with respect to the dilute limit even at $x>2 R_{\mathrm{h}}^{\mathrm{o}}$ (see Figure 3 ). The interpenetration of these solvophilic tails is clearly visible in the bottom right panels of Figure 2, where the density profiles from the two concentration gradients computations in either the radial or the longitudinal length is of the order of $2 R_{\mathrm{h}}^{\mathrm{o}}$.

We use the micelle size in the dilute limit as characteristic length scale for the pair interaction between micelles. The variation of the aggregation number as a function of the normalized intermicelle distance $\tilde{x}=x /\left(2 R_{\mathrm{h}}^{\mathrm{o}}\right)$ is shown in Figure 3. Because of the lattice-nature of the approach followed, it is useful to compare the aggregation number change $(\Delta g)$ normalized by the number of nearest neighboring micelles $K$ when bringing the micelles closer to each other 


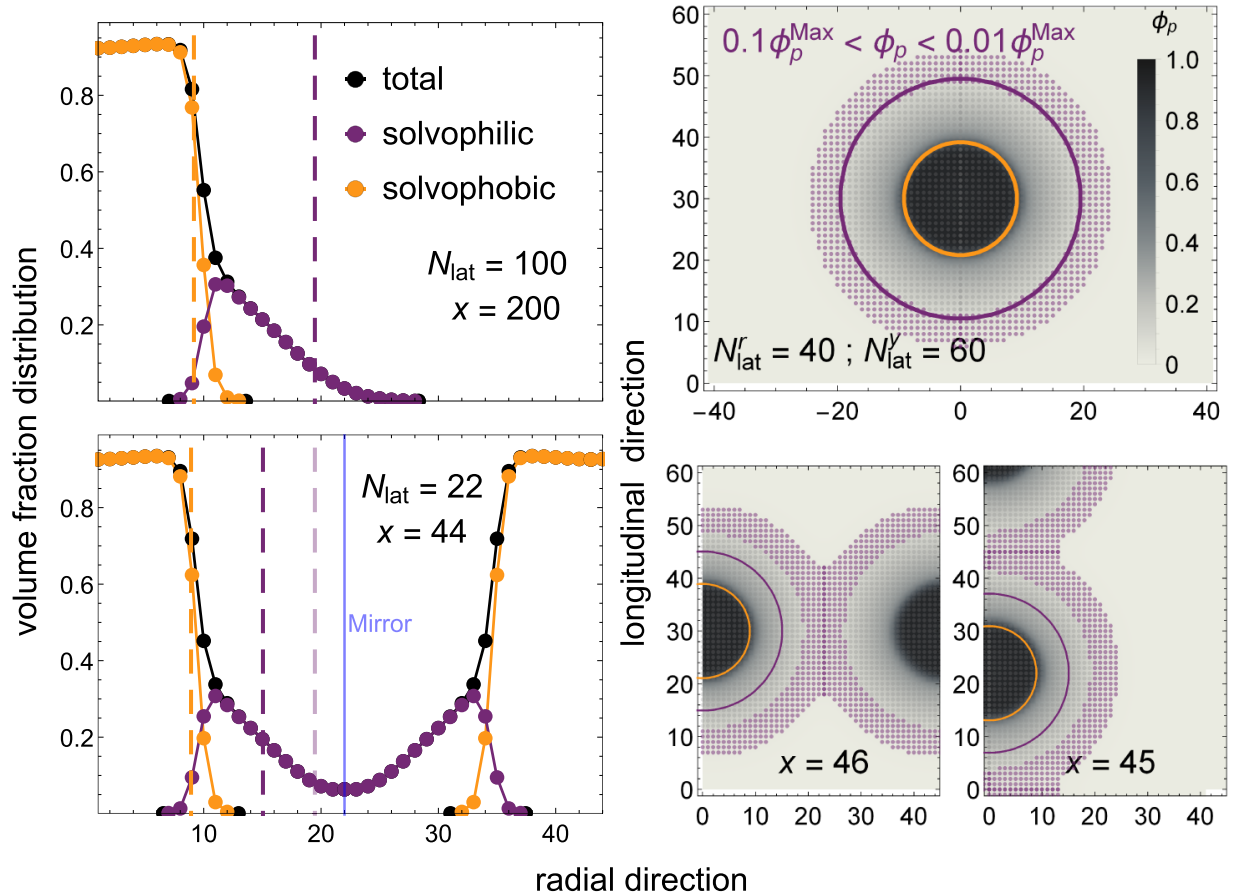

Figure 2. Concentration profiles computed using SCF theory in a spherical lattice with concentration gradients in one dimension (left panels) or in a cylindrical lattice with concentration gradients in two dimensions (right panels) at equilibrium conditions (see Figure 1). Nearest-neighbor micelle distances $x$ are indicated, as well as the number of lattice sites $\left(N_{\text {lat }}\right)$. Vertical lines correspond either to the corresponding hydrodynamic sizes (orange and purple) or to the mirror (blue, only in the bottom leftmost panel). Orange and purple circles (right panels) correspond to the sizes on the left, while clouds of purple points correspond to polymer concentrations in between 1 and $10 \%$.

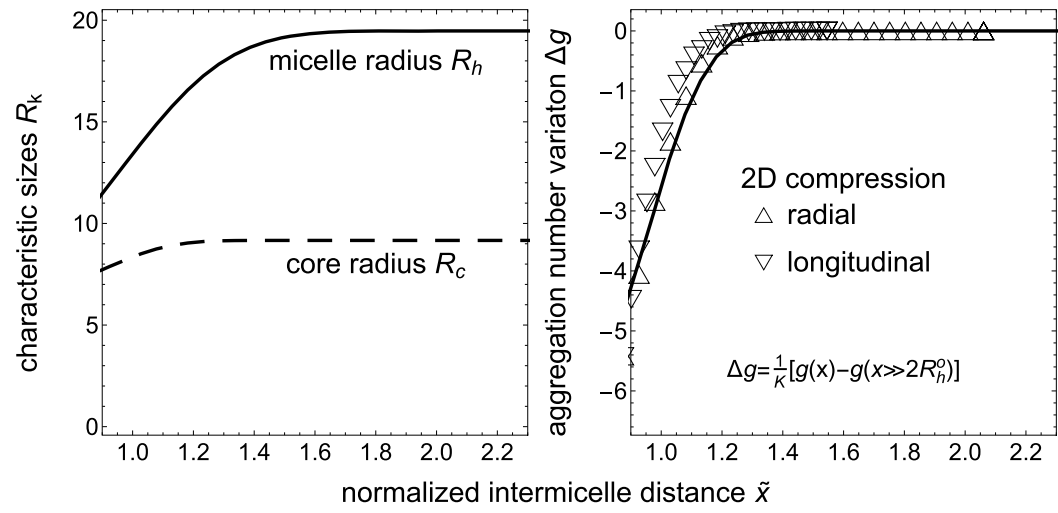

Figure 3. Characteristic size and effective change of aggregation number as a function of the normalized intermicelle distance $\tilde{x}=x / 2 R_{\mathrm{h}}^{\circ}$ for the same system parameters as in Figure 1.

$$
\Delta g_{\mathrm{p}}=\frac{1}{K}\left[g_{\mathrm{p}}(\tilde{x})-g_{\mathrm{p}}(\tilde{x} \gg 1)\right]
$$

As micelles get closer (decreasing $x$ ), their sizes decrease because of the overlap of the solvophilic tails, which leads to contraction of the coronas. From the results in Figure 3, it follows that both the characteristic size of the micelle core and corona as well as the aggregation number decrease upon bringing micelles closer together. The overall size decreases already for $x \lesssim 3.2 R_{\mathrm{h}}^{\mathrm{o}}$ : the outer solvophilic tails start to interact significantly near $\tilde{x} \approx 1.6$. The onset of the decrease of the core size and aggregation number appear simultaneously near $\tilde{x} \approx$ 1.2. This may be explained by the strong dependence of the aggregation number on the core and corona-forming block size: ${ }^{35}$ for $\tilde{x} \lesssim 1.2$, the core is compressed due to the fact that diblock copolymers start to dissociate from the micelle.
The decrease of the aggregation number of the micelles as they get closer (see Figure 3) contrasts with regular scaling models, where the aggregation number is assumed to remain constant up to the limit where micelles overlap and increases beyond overlap of the micelles. ${ }^{38}$ We note that the models presented here concern dilute suspensions of micelles, as we do not study the micellar changes for $r<2 R_{\mathrm{h}}^{\mathrm{o}}$. Upon approaching overlap of micelles, a small decrease of the aggregation number (of the order of what we find here) has been found experimentally. ${ }^{38}$ The trend shown in Figure 3 holds when increasing the solvophilic block length (see the Supporting Information) but the variation in $g_{\mathrm{p}}$ is smaller with increasing $n$ at fixed $\tilde{x}$ (as micelles become more star-like). It has been suggested that the increase of $g_{\mathrm{p}}$ above overlap of the micelles is associated with a change of the preferred micellar morphology far beyond micelle overlap concentration. ${ }^{22,23}$ 
The 2D-gradient SCF approach followed here might be able of capturing such effects, which are out of the scope of the present study.

Model Comparison and Lattice Geometry Effects. In this section, the pair potentials obtained via the different theoretical approaches and using different lattice types are compared. Again, we consider diblock copolymers $\mathrm{B}_{24} \mathrm{~A}_{45}$ with parameters $\chi_{\mathrm{BW}}=2, \chi_{\mathrm{AW}}=0.4, \chi_{\mathrm{AB}}=1$. The dependence of the micelle equilibrium with intermicelle distance provide all required components for calculating the pair interactions (see Methods), which are presented in Figure 4. We consider purely

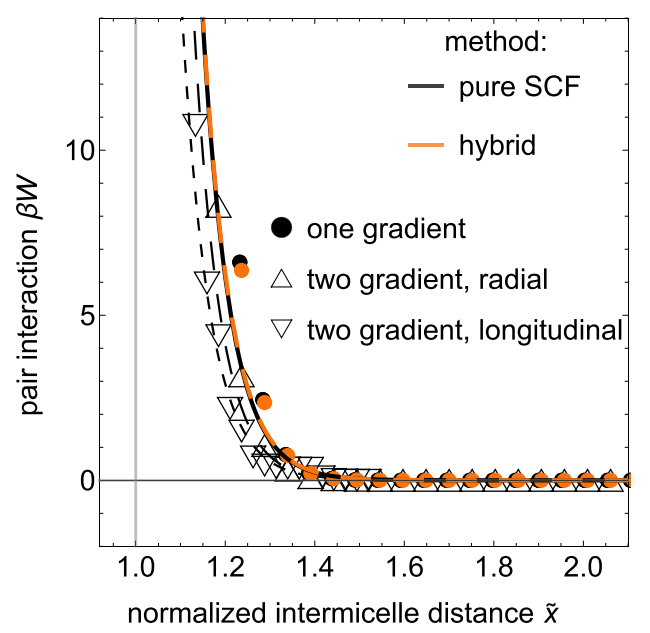

Figure 4. Interaction potential considering different pure SCF approaches and the hybrid model (eq 8), where the values of $g_{\mathrm{p}}$ are obtained from SCF. Curves correspond to the hard-core-Yukawa (HCY) potential fit while symbols are calculated points. Solid, black curve correspond to the $\mathrm{HCY}$ fit of the one concentration gradient calculations. Dashed, orange curve corresponds to the hybrid method. Dashed curves hold for the two-gradient computations where micelles are brought closer to each other in the radial or longitudinal direction.

SCF lattice computations using one or two concentration gradients, as well as an analytical approach (see Methods) in which the only input from the SCF computations is the change in the aggregation number as a function of the intermicelle distance (denoted as Hybrid). The different methods produce very similar results: a strong, short-ranged repulsion takes place at short intermicelle distances $(\tilde{x} \lesssim 1.4)$ which originates from the excluded volume repulsion of the solvophilic tails, corresponding to the situation where coronas contract (see left panel of Figure 3). This repulsive interaction is similar to a brush-like repulsion between polymer-grafted colloids ${ }^{12,13,39}$ and star-like polymers. ${ }^{40}$ For spherical micelles, however, we find that the "surface" at which the "brushes" (solvophilic tails) are grafted is soft, and the effective grafting density is dynamic: both $g_{\mathrm{p}}$ and $R_{\mathrm{h}}$ depend on the distance between the micelles. SCF accounts for the soft and dynamic nature of the micelles.

In Figure 4, the calculated interaction potential from SCF and the hybrid approach are plotted. The interactions between two micelles using the different approaches are quite close. It appeared to be convenient to fit the interaction potential via a HCY interaction. This allows to systematically quantify the range of repulsion $\left(q_{\mathrm{Y}}\right)$ between micelles and how the interaction depends on the diblock copolymer properties. Further, the HCY model has been proposed as a model potential for the interaction between block copolymer micelles. ${ }^{41}$ The fitting results (details can be found in the
Supporting Information) are presented in Table 1. The fitted HCY curves can describe the SCF data points quite well, see

Table 1. Relative Range of the Interaction $q_{\mathrm{Y}}$ and Normalized Second Virial Coefficient $B_{2}^{*}$ of the Example Pair Potentials Presented in Figure $4^{a}$

\begin{tabular}{llcc}
\multicolumn{1}{c}{ method } & $K$ & $q_{\mathrm{Y}}$ & $B_{2}^{*}$ \\
1D SCF & 12 & 0.13 & 9.9 \\
eq 8 + SCF (hybrid) & 12 & 0.13 & 9.9 \\
2D SCF radial compression & 6 & 0.12 & 9.2 \\
2D SCF longitudinal compression & 2 & 0.13 & 8.9 \\
${ }^{a}$ Diblock copolymer characteristics: & $\mathrm{B}_{24} \mathrm{~A}_{45}, \chi_{\mathrm{BW}}=2, \chi_{\mathrm{AW}}=0.4$, and \\
$\chi_{\mathrm{AB}}=1$.
\end{tabular}

Figure 4. Variations are expected in the contact potential values for the different approaches due to the steepness of the interactions calculated. The $q_{\mathrm{Y}}$ values obtained do however not vary significantly. The small differences can be related to slightly different $\Delta g_{\mathrm{p}}(x)$ values, see Figure 3. Our bottom-up approach differs from the ones previously reported in literature, where the mapping of the micelle-micelle repulsion into a $\mathrm{HCY}^{41}$ or pure hard sphere ${ }^{42}$ pair potential was performed using a top-down approach, via fitting experimentally collected structure factors with theoretical ones.

To gain more insights into the colloidal stability of micelle suspensions, we also compare the obtained normalized second virial coefficient $B_{2}^{*}=B_{2} / v_{\mathcal{c}}$, where the effective colloidal particle volume $v_{c}$ is taken as the hydrodynamic volume of a micelle in the dilute solution limit

$$
v_{\mathrm{c}}=\frac{4 \pi}{3}\left(R_{\mathrm{h}}^{\mathrm{o}}\right)^{3}
$$

We summarized the results for the potentials and $B_{2}^{*}$ in Figure 4 in Table 1. Details on the calculation of $B_{2}^{*}$ from the pair interaction can be found in the Supporting Information. The slight decrease of $B_{2}^{*}$ with decreasing the number of nearest-neighbor micelles $K$ points toward a small overestimation of the contact potential values when calculations on the spherical lattice are conducted, most likely because of the different core compressions induced. Deviations of the results depending on the number of concentration gradients using SCF computations are expected. ${ }^{13,43}$ However, SCF calculations in the spherical lattice are sufficiently accurate to resolve the main characteristics of diblock copolymer micellemicelle interactions.

Coronal Solvency Effects. Next, we discuss the effects of the solvophilic block solvency parameter $\left(\chi_{\mathrm{AW}}\right)$ on the intermicellar interactions of the same block copolymer type as before $\left(\mathrm{B}_{24} \mathrm{~A}_{45}\right.$ with $\chi_{\mathrm{BW}}=2$ and varying $\left.\chi_{\mathrm{AW}}\right)$. This solvency parameter governs the colloidal stability of the micellar suspension (as shown in the next section). We consider SCF computations with concentration gradients in one direction. In Figure 5, results are shown for the pair interaction for several $\chi_{\mathrm{AW}}$ values. The black curve corresponds to $\chi_{\mathrm{AW}}=0.4$, the reference situation reported already in the previous section. For $\chi_{\mathrm{AW}}<0.4$, the repulsions get more long-ranged which increases $B_{2}^{*}$ (see also Table 2). By increasing the solvent quality for the corona-forming blocks, the tails extend further from $R_{\mathrm{h}}^{\mathrm{o}}$. This leads to a longer-ranged repulsion. The opposite trend is observed for $\chi_{\mathrm{AW}}>0.4$. Strikingly, a shallow attraction between the micelles around $x=1.2$ appears for $\chi_{\mathrm{AW}}=0.5$. At the $\Theta$-solvent conditions, the excluded volume between 


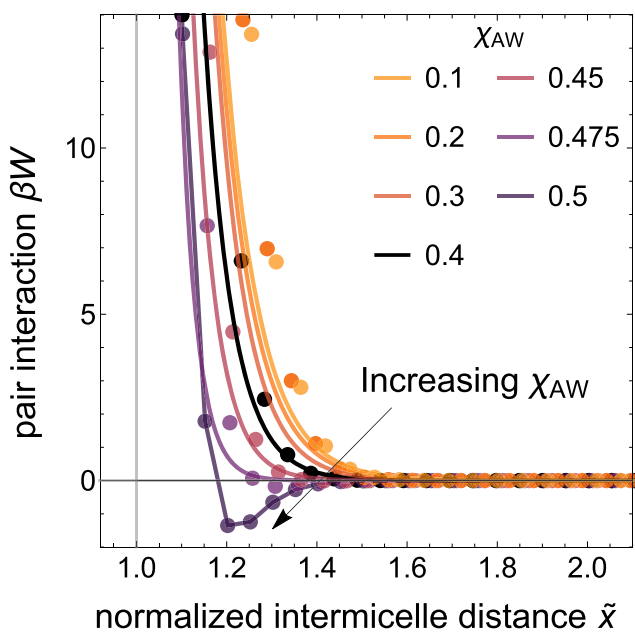

Figure 5. Interaction potential between micelles for various solvent quality parameters of the corona-forming block. Diblock copoylmer considered is $\mathrm{B}_{24} \mathrm{~A}_{45}, \chi_{\mathrm{BW}}=2, \chi_{\mathrm{AB}}=1$, and varying $\chi_{\mathrm{AW}}$ as indicated. Curves correspond to the HCY potential fit while symbols are extracted from SCF data. For $\chi_{\mathrm{AW}}=0.5$, the calculated points are simply joined as a HCY fit is not applicable in this case.

Table 2. Range of Repulsion and Normalized Second Virial Coefficient for a Collection of Pair Potentials Using the Spherical Lattice via the SCF Approach and the Hybrid Approach

\begin{tabular}{lcccc}
$\chi_{\mathrm{AW}}$ & $q_{\mathrm{Y}}^{\text {SCF-1D }}$ & $B_{2}^{*, \text { SCF-1D }}$ & $q_{\mathrm{Y}}^{\mathrm{HYB}}$ & $B_{2}^{*, \mathrm{HYB}}$ \\
0.5 & $\mathrm{n} / \mathrm{a}$ & 1.0 & $\mathrm{n} / \mathrm{a}$ & 1.6 \\
0.475 & 0.08 & 7.1 & 0.08 & 7.1 \\
0.45 & 0.11 & 8.6 & 0.11 & 8.6 \\
0.4 & 0.13 & 9.9 & 0.13 & 9.9 \\
0.3 & 0.14 & 11.0 & 0.14 & 11.0 \\
0.2 & 0.16 & 11.8 & 0.16 & 11.9 \\
0.1 & 0.17 & 12.3 & 0.17 & 12.4 \\
\hline
\end{tabular}

corona-forming segments is exactly compensated by the attraction among them. When the corona blocks start to overlap, these attractions become increasingly important as there are less corona-solvent contacts. This explains, we think, the attractive part of the potential for $\chi_{\mathrm{AW}}=0.5$. The repulsion contribution at $\Theta$-solvent conditions arises from compression of the core. Upon further increase, $\chi_{\mathrm{AW}}$ the attractive part of the potential would increase.

In Table 2, we present the obtained range of repulsion (when possible) as well as the normalized second virial coefficient for the potentials in Figure 5. With increasing $\chi_{\mathrm{AW}}$, the $q_{\mathrm{Y}}$ values get smaller and $B_{2}^{*}$ decreases. Near $\chi_{\mathrm{AW}} \approx 0.5$, the colloidal stability of the micelle suspension drops strongly. The pair interactions (hence their fitting parameters) do not significantly vary with the method (hybrid method or pure SCF) used in their calculation.

Solvophilic Block Length Effects. In this section, we address the effect of varying the solvophilic block length, which leads to an increase of the coronal thickness. The effect of increasing the coronal thickness is twofold. On the one hand, because of a soft decay of the coronal domain (concentration profiles in the Supporting Information), the steric repulsion gets more long-ranged (reflected in a larger $q_{\mathrm{Y}}$ value, see Table 3 ). On the other hand, the aggregation number decreases whilst increasing the hydrodynamic size because of an increased overall diblock solvency (see the Supporting
Table 3. HYC-Fitted Range of Interaction, Extrapolated Contact Potential (from the Data-Points), and Normalized Second Virial Coefficient for a Collection of Pair Potentials Obtained from the SCF Approach and Using Eq 8, Where the Values of $g_{p}$ are Calculated with the SCF Theory for Micelle Formed of Block $\mathrm{B}_{24} \mathrm{~A}_{n}{ }^{a}$

$\begin{array}{lcccccc}n & q_{\mathrm{Y}}^{\text {SCF-1D }} & \epsilon^{\text {SCF-1D }} & B_{2}^{*, S C F-1 D} & q_{\mathrm{Y}}^{\mathrm{HYB}} & \epsilon^{\mathrm{HYB}-1 \mathrm{D}} & B_{2}^{*, \mathrm{HYB}} \\ 45 & 0.128 & 91.6 & 9.8 & 0.128 & 87.5 & 9.8 \\ 90 & 0.148 & 48.5 & 9.8 & 0.148 & 48.6 & 9.8 \\ 135 & 0.161 & 33.0 & 9.8 & 0.161 & 34.2 & 9.9 \\ 180 & 0.165 & 25.1 & 9.4 & 0.164 & 26.7 & 9.5 \\ 225 & 0.167 & 21.8 & 9.2 & 0.166 & 23.8 & 9.4 \\ 270 & 0.173 & 18.3 & 9.2 & 0.172 & 20.4 & 9.4 \\ 315 & 0.177 & 15.3 & 9.0 & 0.176 & 17.3 & 9.2 \\ 360 & 0.179 & 13.2 & 8.8 & 0.178 & 15.2 & 9.0 \\ 405 & 0.179 & 12.0 & 8.6 & 0.178 & 13.9 & 8.8 \\ 450 & 0.180 & 11.1 & 8.4 & 0.178 & 13.1 & 8.7\end{array}$

$a_{\text {The }} \mathrm{FH}$ interaction parameters are $\chi_{\mathrm{BW}}=2, \chi_{\mathrm{AW}}=0.4$ and $\chi_{\mathrm{AB}}=1$.

Information for details on the dependence of $g_{\mathrm{p}}$ with diblock properties). Thus, the effective grafting density of solvophilic tails from the core (see next section) decreases with increasing $n$. As observed in the previous section, the strongest contribution to the steric repulsion between micelles arises, within our model, from compression of the core. Thus, there is a balance between the range and the strength of the steric repulsion because of the coronal decays with increasing the solvophilic block length, which leads to an overall high $B_{2}^{*}$ value, which weakly depends on the particular $n$-value. When increasing $n$, the decrease of $g_{\mathrm{p}}$ weakens (see the Supporting Information): the core compression gets more screened upon increasing the corona thickness.

In Figure 6, examples of pair potentials for various $n$-values $\left(\mathrm{B}_{24} \mathrm{~A}_{n}\right.$ with $\chi_{\mathrm{BW}}=2, \chi_{\mathrm{AW}}=0.4$, and $\chi_{\mathrm{AB}}=1$; black curve corresponds to the chosen reference diblock) are plotted. The shape of the interaction potentials resemble those presented in Figure 4. As can be appreciated, the HCY potential fits even better for larger $n$-values: the interaction between diblock copolymeric micelles (particularly, with long hydrophilic tails)

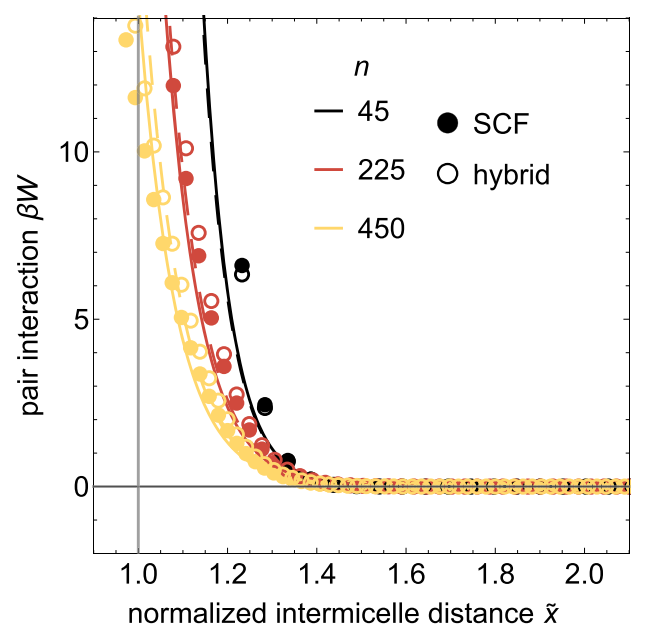

Figure 6. Interaction potential between micelles for various solvophilic block lengths $n$ as indicated. The diblock copolymer considered is $\mathrm{B}_{24} \mathrm{~A}_{n}, \chi_{\mathrm{BW}}=2, \chi_{\mathrm{AB}}=1$, and $\chi_{\mathrm{AW}}=0.4$. Solid curves correspond to the HCY potential fit of the SCF data, whilst dashed curves correspond to fittings of the hybrid approach presented. 

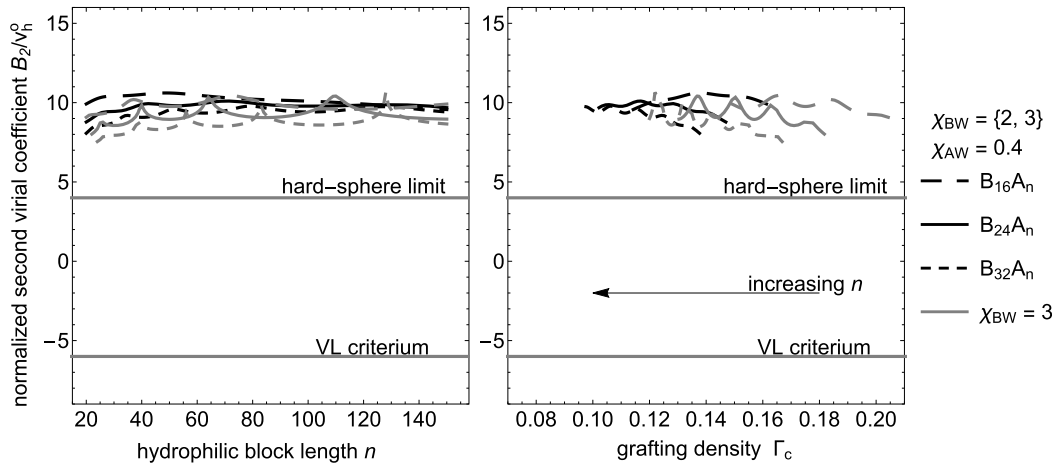

Figure 7. Left panel: Normalized second virial coefficient $B_{2}^{*}$ of spherical micelles composed of diblock copolymers $B_{24} A_{n}$ with increasing solvophilic block length $n$. Right panel: $B_{2}^{*}$ for the same data sets as on the left panel but in terms of the grafting density of solvophilic segments from the core $\Gamma_{c}$.

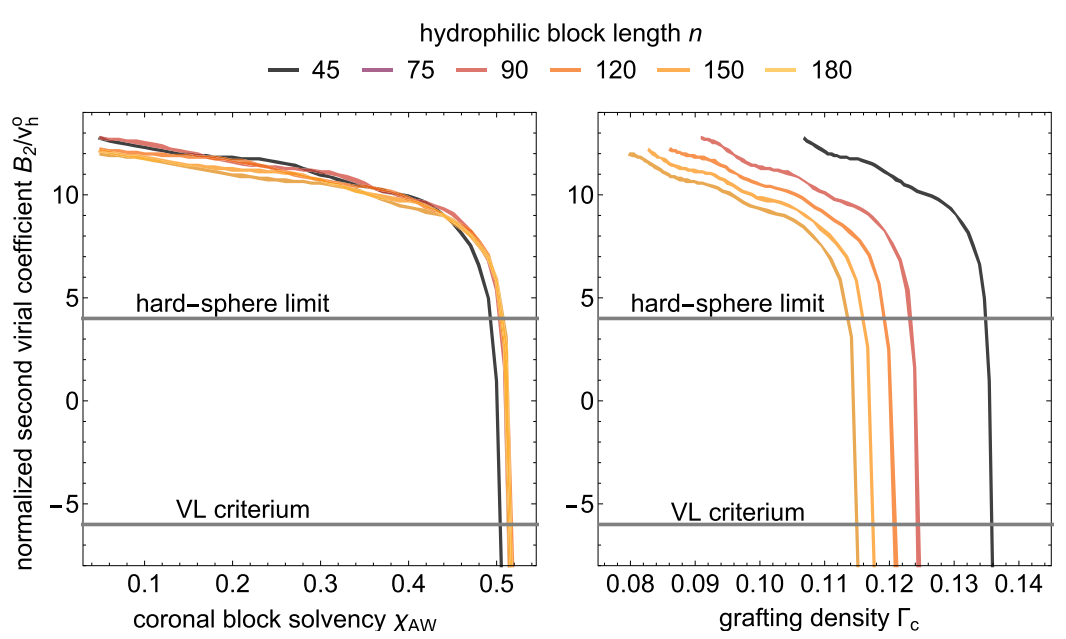

Figure 8. Left panel: Influence of the coronal block solvency (via $\chi_{\mathrm{AW}}$ ) on the normalized second virial coefficient $B_{2}^{*}$ of spherical micelles composed of diblock copolymers $B_{24} A_{n}$ for a collection of different solvophilic block lengths $n$. Right panel: $B_{2}^{*}$ for the same data sets as on the left but in terms of the grafting density of solvophilic segments at the core-corona interface.

is similar to the outer-soft-core contribution of the interaction between star-like polymers. ${ }^{19,21}$ In Table 3, the resulting HCYfitted interaction range, contact potential, and normalized second virial coefficient are listed for various hydrophilic block lengths $n$. For the conditions investigated, $B_{2}^{*}$ still remains approximately constant (though a slight decrease is appreciated when considering very large coronal domains).

On the Colloidal Stability of Spherical Micelles. In this section, a colloidal stability overview of diblock copolymer micelle suspensions is presented in terms of the calculated second virial coefficient. This quantity can be related to the colloidal stability and can be experimentally measured using light-scattering techniques. The interaction between polymer brushes anchored to solid surfaces (steric stabilization) sensitively depends on the grafting density of polymers. ${ }^{12,44,45}$ To compare the interactions between colloidal spheres with anchored polymeric brushes and those between spherical copolymer micelles, we considered an effective grafting density of solvophilic blocks at the core-corona interface

$$
\Gamma_{\mathrm{c}}=\frac{g_{\mathrm{p}}}{4 \pi R_{\mathrm{c}}^{2}}
$$

where $R_{c}$ is the core size, estimated from the SCF concentration profiles. The absolute value of $\Gamma_{c}$ for diblock copolymer micelles depends on the considered system parameters: the number of lyophobic block segments $(m)$, the number of lyophilic block segments $(n)$, and their solvency parameters $\left(\chi_{\mathrm{BW}}\right.$ and $\left.\chi_{\mathrm{AW}}\right)$. However, in terms of the colloidal stability, the solvency and length of the core-forming blocks ( $m$ and $\chi_{\mathrm{BW}}$ ) does hardly affect $B_{2}^{*}$ (as shown in the left panel of Figure 7) for the spherical micelles studied. This is due to a balance between the range (increasing with $n$ ) and the strength (decreasing with $n$ ) of the steric repulsion (details in the Supporting Information). In fact, a value of $B_{2}^{*} \approx 9 \pm 1$ is found independent of $\left\{m, n, \chi_{\mathrm{BW}}\right\}$ for a fixed coronal block solvency of $\chi_{\mathrm{AW}}=0.4$ (see Figure 7 ). Hence, the grafting density at the core-corona interface hardly mediates repulsive micelle-micelle interactions. This is in contrast with the expectations for the interaction between hard spheres with anchored brushes. Next, we focus again on the influence of the interaction between the solvophilic tails as mediated by $\chi_{\mathrm{AW}}$.

The influence of the corona block solvency $\left(\chi_{\mathrm{AW}}\right)$ on $B_{2}^{*}$ is plotted in Figure 8 . For $\chi_{\mathrm{AW}} \leq 0.45$, we find $B_{2}^{*}>4$ for all values of $n$, indicating that micelles with these characteristics always interact in an overall (highly) repulsive fashion. The value of $\chi_{\mathrm{AW}}$ does not only affect the grafting density but also the interaction between the coronal tails. Upon approaching $\Theta$-solvent conditions $\left(\chi_{\mathrm{AW}} \rightarrow 0.5\right)$, the mutual excluded volume repulsion decreases. Thus, and contrary to what is expected from sterically stabilized inorganic colloids, ${ }^{12,44} B_{2}^{*}$ 
decreases because of solvophobic effects with increasing the (diblock properties dependent) grafting density.

The attractive part of the pair interaction may be deep enough to destabilize the micelle suspension (Figure 5), as seen in Figure 8 (left panel) for $\chi_{\mathrm{AW}} \gtrsim 0.5$. In contrast with theoretical predictions for polymer-grafted colloids, ${ }^{12,44}$ this colloidal destabilization arises (within our model) without considered direct attractions between micelles. Colloidal destabilization around $\Theta$-solvent conditions for the corona arises because of solvophobic effects: the enthalpic gain due to the solvent expel as micelles get closer is sufficient to compensate the entropic penalty of compressing the solvophilic tails.

\section{CONCLUSIONS}

In this paper, the interaction between dilute (diblock) copolymer micelles is quantified using numerical SCF computations and analytical theory. We use the aggregation number obtained from the SCF computations as an input for the analytical theory. The intermicelle pair-potentials obtained via the two methods are in good agreement, also when considering different lattice topologies. Particularly, the range of the interaction and the normalized second virial coefficient are all rather similar: they are not sensitive to the method used and to how many concentration gradients are considered. In our approach, we account for the soft and dynamic nature of these association colloids as the micelles get closer. At each condition, the equilibrium micellization is re-evaluated: all polymer blocks in the micelles remain associative and fully responsive and can conformationally rearrange and equilibrate at each condition. For coronal domains whose solvency is better than $\Theta$-solvent conditions, this results in a HCY-like repulsion for all cases studied. The range of this repulsion depends on the solvophilic block length, whereas its strength decreases with increasing solvophilic block chain because of a decrease of their effective grafting density, which leads to a weak dependence of the second virial coefficient on solvophilic block length.

We find that the colloidal stability of a dilute diblock copolymer micelle suspension is only weakly affected by the nature of the core (solvophobicity and chain length of the core blocks). Not surprisingly, colloidal suspensions of diblock copolymer micelles are always stable (normalized second virial coefficient $\left.B_{2}^{*}>-6\right)$ unless the solvophilic blocks are near $\Theta$ solvent or in poor solvent conditions. For fixed core-forming block properties but different solvophilic block length, the normalized second virial coefficients with varying coronal block solvency follow a similar curve. Furthermore, and contrary to what is expected from polymer-grafted colloidal particles, increasing the effective grafting density of solvophilic blocks from the micelle core decreases the colloidal stability of the micellar suspension. This is explained because of the interplay between $\Gamma_{c}$ and the properties of the solvophilic and solvophobic blocks. The SCF method presented here for intermicelle interactions can be extended to account for more components in solution and other block sequences.

\section{METHODS}

In this section, we summarize the theoretical and numerical methods used to study the diblock copolymer micelles.

SCF for Micelle-Micelle Interactions. We used the Scheutjens-Fleer self-consistent lattice theory $(\mathrm{SCF})^{46,47}$ to perform numerical calculations. It is based upon FloryHuggins mean-field theory, ${ }^{48}$ but with concentration gradients following discrete versions of the Edwards equation accounting for the mean-field polymer propagation. ${ }^{49}$ The interdependence of the segment potentials $\left(u_{k}\right)$ and the volume fractions $\left(\phi_{k}\right)$ for each component in the system

$$
\phi_{k}\left(u_{k}\right) \leftrightarrow u_{k}\left(\phi_{k}\right)
$$

is the core idea of the self-consistent method. ${ }^{50}$ Provided a user-defined starting configuration for the components in the lattice, the free energy of the lattice is minimized in a selfconsistent fashion. The boundary conditions and the lattice geometry need to be specified. We consider here two different lattice types, namely, a spherical lattice with concentration gradients in one direction and a cylindrical lattice with concentration gradients in two directions. Mirror boundary conditions are set for all boundaries. ${ }^{51} \mathrm{~A}$ spherical lattice is defined as shells from the center $(r=0)$ of the lattice up to $r=$ $N_{\text {lat }}$. The first lattice layer corresponds to the center of the spherical micelle. As we focus on conditions where a spherical micelle is preferred over other self-assembled structures, a spherical lattice is used in most of our calculations. A cylindrical lattice is defined by a grid of $N_{\text {lat }}^{r}$ sites in the radial coordinate and $N_{\text {lat }}^{\mathrm{y}}$ sites in the longitudinal coordinate, and we use it here simply to asses the validity of the calculation of the pair potential using the spherical lattice.

Because of the mirror conditions imposed, a micelle is formed in the presence of $K$ surrounding ones. The distance between the centers of two nearest-neighbor micelles $x$ defines the characteristic length scale involved in the pair potential calculation. For the spherical lattice, $K=12$ and $x=2 N_{\text {lat. }}$. For the cylindrical one, six cylindrical lattices are present around the simulated one in the radial direction $\left(K_{\mathrm{r}}=6\right)$ while two span from the top and bottom of the radial mirror conditions [one from the upper and one from the lower boundaries of the lattice, $\left.\left(K_{\mathrm{y}}=2\right)\right]$. In this case, the pair-potential calculation depends on how $N_{\text {lat }}^{(\mathrm{k})}$ is varied $(\mathrm{k}=\{\mathrm{r}, \mathrm{y}\})$. In the cylindrical lattice, micelles are formed in the center of the radial axis of symmetry (see Figure 2). This implies that the nearest neighbors are at distances $x_{\mathrm{r}}=2 N_{\mathrm{lat}}^{\mathrm{r}}$ (radial direction) and $x_{\mathrm{y}}=$ $N_{\text {lat }}^{\mathrm{y}}$ (longitudinal direction).

The SCF approach is combined with small system thermodynamics ${ }^{52}$ to study the conditions under which the diblock copolymers form self-assembled morphologies. ${ }^{53}$ To find the equilibrium configuration, we compute the grand potential $\Omega$ of the system for a specific diblock copolymer as a function of the aggregation number $g_{\mathrm{p}}$. This grand potential relates to the inhomogeneities in the system: in a pure solvent $\Omega=0$. As diblock copolymers are added to the solution, $\Omega$ increases because of the contacts present between solution and solvophobic blocks. The appearance of the first thermodynamically stable micelle is marked by a maximum in $\Omega$. If a micelle can form $\Omega$ decreases with $g_{\mathrm{p}}$, and at a given diblock concentration the condition $\Omega_{g \neq 0}=0$ is met (with $\partial \Omega / \partial g_{\mathrm{p}}<$ $0)$. At this condition, the block copolymers in the micelle are in equilibrium with free block copolymers in the bulk: the chemical potential of one copolymer in the micelle is equal to that in the bulk

$$
\mu_{\mathrm{p}}^{\text {bulk }}=\mu_{\mathrm{p}}^{\text {micelle }} \equiv \mu_{\mathrm{p}} \approx k_{\mathrm{B}} T \ln \phi_{\mathrm{p}}^{\text {bulk }}
$$

with $\phi_{\mathrm{p}}^{\text {bulk }}$ being the bulk polymer segment volume fraction. Once an equilibrium micelle is found, we characterize its size 
by calculating the hydrodynamic radius ${ }^{54}$ (see the Supporting Information). The work required to dissociate all polymers from the fully grown self-assembled structure (equivalently, the energy gain of the diblocks upon micellization) at a certain intermicelle distance $x$ follows as ${ }^{55}$

$$
\omega(x)=g_{\mathrm{p}}(x) \mu_{\mathrm{p}}(x)+g_{\mathrm{s}}(x) \mu_{\mathrm{s}}(x)
$$

where $g_{s}$ is the excess number of solvent monomers (provided by SCF). The chemical potential for the solvent is evaluated at the micelle equilibrium condition via

$$
\mu_{\mathrm{s}} \approx k_{\mathrm{B}} T \ln \phi_{\mathrm{s}}^{\text {bulk }}
$$

with $\phi_{\mathrm{s}}^{\text {bulk }}$ the bulk concentration of solvent. Even at small intermicelle distances $x$, there is always a high volume fraction of solvent in the bulk as considered in SCF. Hence, $\ln \phi_{\mathrm{s}}^{\text {bulk }} \approx$ 0 , and $w(x)$ is dominated by the changes in the diblock copolymer as expected (see the Supporting Information). These SCF-provided equilibrium quantities enable us to estimate the pair interaction potential between micelles as

$$
W(x)=\frac{2}{K}[\omega(x)-\omega(x=\infty)]
$$

Thus, the equilibrium properties computed from SCF provide a route for calculating the pair potentials considering a micelle composed of $g_{\mathrm{p}}$ polymers surrounded by $K$ other micelles at a distance $x$. Our approach does not imply any ad hoc interaction between the coronas. We extract pair interactions between the micelles, which originate naturally from the equilibrium properties of micelles formed at aiven $x$. For small enough $x$, the coronas start to overlap (see Figure 2), affecting the equilibrium micelle formation conditions and hence the free energy of micelle formation.

Analytical Expression for the Interaction Potential. An analytical expression for the interaction potential can be obtained from previously developed theories for block copolymer micelles. The presence of the $K$ surrounding micelles exerts an isotropic compression on the central micelle for values of $r$ smaller than, say, the micelle's undistorted (dilute limit) diameter $2 R_{\mathrm{h}}^{\mathrm{o}}$. The confined micelle is assumed to be in equilibrium with free copolymer molecules, which enables to minimize the unfavorable increase of the free energy upon compression; the aggregation number $g_{\mathrm{p}}$ is allowed to vary with $x$. The pair interaction potential $W(x)$ can be expressed as

$$
W(x)=\frac{2}{K}\left[f_{\text {mic }}(x)-f_{\text {mic }}(x=\infty)\right]
$$

where $f_{\text {mic }}(x)$ is the free energy of a micelle whose center is separated by a distance $x$ from a neighboring one. Hence, $f_{\text {mic }}(x=\infty)$ is the free energy of an isolated micelle. The free energy of a micelle can be approximated as the sum of three contributions: the elastic free energy of the core-forming blocks, the elastic free energy of the corona-forming blocks, and the interfacial energy between the core and the solvent at the core-corona interface. We use an approximate expression for $f_{\text {mic }}(x)=\frac{F_{\text {mic }}(x)}{k_{\mathrm{B}} T}$ by modifying a result from Zhulina and Borisov $^{35}$

$$
\begin{aligned}
f_{\text {mic }}(x)= & \frac{g_{\mathrm{p}}(x) \frac{3 \pi^{2} R_{\mathrm{c}}^{2}(x)}{80 l^{2} m}}{\text { elastic, core }}+\frac{\frac{g_{\mathrm{p}}(x)^{3 / 2}}{2 \sqrt{\pi}} \ln \left[1+\frac{T(x)}{R_{\mathrm{c}}(x)}\right]}{\text { elastic, corona }} \\
& +\frac{\frac{4 \pi \gamma\left[R_{\mathrm{c}}(x)^{2}-g_{\mathrm{p}}(x) R_{\mathrm{B}}{ }^{2}\right]}{\text { core-corona interface }}}{}
\end{aligned}
$$

Here, $l$ is the size of a monomer, $R_{c}(x)$ and $T(x)$ represent the core radius and the corona thickness, respectively, $R_{\mathrm{B}}$ is the radius of the collapsed $B$ block in an unassembled block copolymer molecule, and $\gamma$ is the interfacial tension between the core and the solvent. The value of $\gamma$ is calculated approximately from the Helfand-Tagami equation ${ }^{56}$

$$
\gamma=\sqrt{\chi_{\mathrm{BW}} / 6}
$$

Both $R_{\mathrm{c}}(x)$ and $T(x)$ can be expressed as a function of $g_{\mathrm{p}}(x)$ (see the Supporting Information). Hence, given the $\chi$ parameters and the block copolymer composition, we only need $g_{\mathrm{p}}(x)$ to calculate $W(x)$. Hence, the results of this analytical theory are denoted as hybrid because SCF input is needed (only through $g_{\mathrm{p}}(x)$ ).

System Parameters. Below, the set of system parameters are specified. In general, we use the notation $\mathrm{B}_{m} \mathrm{~A}_{n}$ to denote the diblock whose solvophobic block (B) is composed of $m$ segments and whose solvophilic block (A) is composed of $n$ segments. Next, we specify the interaction of the solvophobic segments $\chi_{\mathrm{BW}}$, the solvophilic segments $\chi_{\mathrm{AW}}$, and the interaction between segments of different nature $\chi_{\mathrm{AB}}$. The set of Flory-Huggins interaction parameters is based upon previous investigations on diblock copolymer systems in water (Table 4$){ }^{36}$

Table 4. Notation-at-Hand and (Fixed) System Parameters

\begin{tabular}{ccccc}
-phobic block length $m$ & -philic block length $n$ & $\chi_{\mathrm{BW}}$ & $\chi_{\mathrm{AW}}$ & $\chi_{\mathrm{AB}}$ \\
\hline $16,24,32$ & from 20 to 450 & 2,3 & varied & 1 \\
\hline
\end{tabular}

We note that even though multiple systematic variations have been conducted, we report only the main results that allowed us to determine how the stability of micelles decreases with effective grafting density of solvophilic chains from the core. Further results of the systematic parameter scan conducted are deferred to the Supporting Information.

\section{ASSOCIATED CONTENT}

\section{Supporting Information}

The Supporting Information is available free of charge on the ACS Publications website at DOI: 10.1021/acsomega.8b02548.

Hydrodynamic size calculation, expressions for $R_{\mathcal{c}}, T$ and $R_{\mathrm{B}}$, model pair potential and second virial coefficient, influence of the solvophilic block length, and further results of the systematic variation (PDF)

\section{AUTHOR INFORMATION}

\section{Corresponding Author}

*E-mail: remco.tuinier@tue.nl, r.tuinier@tue.nl (R.T.).

ORCID

Remco Tuinier: 0000-0002-4096-7107

Author Contributions

${ }^{\S}$ Á.G.G. and A.I. contributed equally to this work. 


\section{Notes}

The authors declare no competing financial interest.

\section{ACKNOWLEDGMENTS}

The authors thank Prof. Frans Leermakers for many useful discussions regarding the SF-SCF calculations. Á.G.G. also thanks Mark Vis and Joeri Opdam for useful comments on the SCF approach for micelle-micelle interaction. We acknowledge NWO, DSM, and SymoChem for funding NWO-TA project 731.015.205.

\section{REFERENCES}

(1) Leermakers, F. A. M.; Eriksson, J. C.; Lyklema, J. In Soft Colloids; Lyklema, J., Ed.; Fundamentals of Interface and Colloid Science; Academic Press, 2005; Vol. 5, pp 4.1-4.123.

(2) Mai, Y.; Eisenberg, A. Self-assembly of block copolymers. Chem. Soc. Rev. 2012, 41, 5969.

(3) Muñoz-Bonilla, A.; Ali, S. I.; del Campo, A.; Fernández-García, M.; van Herk, A. M.; Heuts, J. P. A. Block Copolymer Surfactants in Emulsion Polymerization: Influence of the Miscibility of the Hydrophobic Block on Kinetics, Particle Morphology, and Film Formation. Macromolecules 2011, 44, 4282-4290.

(4) Tuinier, R.; de Kruif, C. G. Stability of casein micelles in milk. J. Chem. Phys. 2002, 117, 1290-1295.

(5) Yang, L.; Qi, X.; Liu, P.; El Ghzaoui, A.; Li, S. Aggregation behavior of self-assembling polylactide/poly(ethylene glycol) micelles for sustained drug delivery. Int. J. Pharm. 2010, 394, 43-49.

(6) Li, W.; Li, J.; Gao, J.; Li, B.; Xia, Y.; Meng, Y.; Yu, Y.; Chen, H.; Dai, J.; Wang, H.; Guo, Y. The fine-tuning of thermosensitive and degradable polymer micelles for enhancing intracellular uptake and drug release in tumors. Biomaterials 2011, 32, 3832-3844.

(7) Wang, J.; Xing, X.; Fang, X.; Zhou, C.; Huang, F.; Wu, Z.; Lou, J.; Liang, W. Cationic amphiphilic drugs self-assemble to the coreshell interface of PEGylated phospholipid micelles and stabilize micellar structure. Philos. Trans. R. Soc., A 2013, 371, 20120309.

(8) Lombardo, D.; Calandra, P.; Barreca, D.; Magazù, S.; Kiselev, M. Soft Interaction in Liposome Nanocarriers for Therapeutic Drug Delivery. Nanomaterials 2016, 6, 125.

(9) Silbert, M.; Canessa, E.; Grimson, M. J.; Scalise, O. H. Sterically stabilized colloidal dispersions: beyond hard spheres. J. Phys.: Condens. Matter 1999, 11, 10119.

(10) Israelachvili, J. N. Intermolecular and Surface Forces, 3rd ed.; Academic Press: San Diego, 2011.

(11) Hamley, I. Block Copolymers in Solution: Fundamentals and Applications; John Wiley and Sons, Ltd., 2005.

(12) Zhulina, E. B.; Borisov, O. V.; Priamitsyn, V. A. Theory of steric stabilization of colloid dispersions by grafted polymers. J. Colloid Interface Sci. 1990, 137, 495-511.

(13) Lin, E. K.; Gast, A. P. Self Consistent Field Calculations of Interactions between Chains Tethered to Spherical Interfaces. Macromolecules 1996, 29, 390-397.

(14) Gast, A. P. Structure, interactions, and dynamics in tethered chain systems. Langmuir 1996, 12, 4060-4067.

(15) Lund, R.; Willner, L.; Richter, D.; Dormidontova, E. E. Equilibrium Chain Exchange Kinetics of Diblock Copolymer Micelles: Tuning and Logarithmic Relaxation. Macromolecules 2006, 39, 45664575.

(16) Choi, S.-H.; Lodge, T. P.; Bates, F. S. Mechanism of Molecular Exchange in Diblock Copolymer Micelles: Hypersensitivity to Core Chain Length. Phys. Rev. Lett. 2010, 104, 047802.

(17) Choi, S.-H.; Bates, F. S.; Lodge, T. P. Molecular Exchange in Ordered Diblock Copolymer Micelles. Macromolecules 2011, 44, 3594-3604.

(18) Lu, J.; Bates, F. S.; Lodge, T. P. Remarkable Effect of Molecular Architecture on Chain Exchange in Triblock Copolymer Micelles. Macromolecules 2015, 48, 2667-2676.

(19) Grason, G. M. Ordered phases of diblock copolymers in selective solvent. J. Chem. Phys. 2007, 126, 114904.
(20) Puaud, F.; Nicolai, T.; Nicol, E.; Benyahia, L.; Brotons, G. Dynamic Arm Exchange Facilitates Crystallization and Jamming of Starlike Polymers by Spontaneous Fine-Tuning of the Number of Arms. Phys. Rev. Lett. 2013, 110, 028302.

(21) Likos, C. N.; Löwen, H.; Watzlawek, M.; Abbas, B.; Jucknischke, O.; Allgaier, J.; Richter, D. Star Polymers Viewed as Ultrasoft Colloidal Particles. Phys. Rev. Lett. 1998, 80, 4450-4453.

(22) Zhulina, E. B.; Adam, M.; LaRue, I.; Sheiko, S. S.; Rubinstein, M. Diblock Copolymer Micelles in a Dilute Solution. Macromolecules 2005, 38, 5330-5351.

(23) Semenov, A. N.; Nyrkova, I. A.; Khokhlov, A. R. Polymers with Strongly Interacting Groups: Theory for Nonspherical Multiplets. Macromolecules 1995, 28, 7491-7500.

(24) Kurnaz, M. L.; Maher, J. V. Measurement of the second virial coefficient for the interaction of dilute colloidal particles in a mixed solvent. Phys. Rev. E: Stat. Phys., Plasmas, Fluids, Relat. Interdiscip. Top. 1997, 55, 572.

(25) Quigley, A.; Williams, D. R. The second virial coefficient as a predictor of protein aggregation propensity: A self-interaction chromatography study. Eur. J. Pharm. Biopharm. 2015, 96, 282-290.

(26) Koňák, C.; Tuzar, Z.; Stěpánek, P.; Sedláček, B.; Kratochvíl, P. Interaction between block copolymer micelles in solution. Frontiers in Polymer Science; Steinkopff-Verlag Heidelberg: Darmstadt, 1985; pp 15-19.

(27) Villacampa, M.; Diaz de Apodaca, E.; Quintana, J. R.; Katime, I. Diblock Copolymer Micelles in Solvent Binary Mixtures. 2. Selective Solvent/Good Solvent. Macromolecules 1995, 28, 4144-4149.

(28) Yoshimura, T.; Esumi, K. Physicochemical properties of anionic triple-chain surfactants in alkaline solutions. J. Colloid Interface Sci. 2004, 276, 450-455.

(29) Li, W.; Nakayama, M.; Akimoto, J.; Okano, T. Effect of block compositions of amphiphilic block copolymers on the physicochemical properties of polymeric micelles. Polymer 2011, 52, 3783-3790.

(30) Zinn, T.; Willner, L.; Lund, R.; Pipich, V.; Appavou, M.-S.; Richter, D. Surfactant or block copolymer micelles? Structural properties of a series of well-defined n-alkyl-PEO micelles in water studied by SANS. Soft Matter 2014, 10, 5212-5220.

(31) Theory and Simulation of Hard-Sphere Fluids and Related Systems; Lecture Notes in Physics; Mulero, A., Ed.; Springer-Verlag, 2008.

(32) Vliegenthart, G. A.; Lekkerkerker, H. N. W. Predicting the gasliquid critical point from the second virial coefficient. J. Chem. Phys. 2000, 112, 5364-5369.

(33) Tuinier, R.; Feenstra, M. S. Second Virial Coefficient at the Critical Point in a Fluid of Colloidal Spheres Plus Depletants. Langmuir 2014, 30, 13121-13124.

(34) Platten, F.; Valadez-Pérez, N. E.; Castañeda-Priego, R.; Egelhaaf, S. U. Extended law of corresponding states for protein solutions. J. Chem. Phys. 2015, 142, 174905.

(35) Zhulina, E. B.; Borisov, O. V. Theory of Block Polymer Micelles: Recent Advances and Current Challenges. Macromolecules 2012, 45, 4429-4440.

(36) Ianiro, A.; Patterson, J.; González García, Á.; van Rijt, M. M. J.; Hendrix, M. M. R. M.; Sommerdijk, N. A. J. M.; Voets, I. K.; Esteves, A. C. C.; Tuinier, R. A roadmap for poly(ethylene oxide)-block -poly$\varepsilon$-caprolactone self-assembly in water: Prediction, synthesis, and characterization. J. Polym. Sci., Part B: Polym. Phys. 2017, 56, 330339.

(37) Guerin, C. B. E.; Szleifer, I. Self-Assembly of Model Nonionic Amphiphilic Molecules. Langmuir 1999, 15, 7901-7911.

(38) Amann, M.; Willner, L.; Stellbrink, J.; Radulescu, A.; Richter, D. Studying the concentration dependence of the aggregation number of a micellar model system by SANS. Soft Matter 2015, 11, 4208-4217.

(39) van Gruijthuijsen, K.; Obiols-Rabasa, M.; Heinen, M.; Nägele, G.; Stradner, A. Sterically Stabilized Colloids with Tunable Repulsions. Langmuir 2013, 29, 11199-11207.

(40) Likos, C. N.; Harreis, H. M. Star polymers: From conformations to interactions to phase diagrams. Condens. Matter Phys. 2002, 5, 173. 
(41) Chen, S.-H.; Broccio, M.; Liu, Y.; Fratini, E.; Baglioni, P. The two-Yukawa model and its applications: the cases of charged proteins and copolymer micellar solutions. J. Appl. Crystallogr. 2007, 40, s321s326.

(42) Brown, G. J.; Richards, R. W.; Heenan, R. K. Organisation and interactions in aqueous dispersions of polystyrene-polyethylene oxide block copolymer micelles. Polymer 2001, 42, 7663-7673.

(43) Bergsma, J.; Leermakers, F. A. M.; van der Gucht, J. Interactions between nodes in a physical gel network of telechelic polymers; self-consistent field calculations beyond the cell model. Phys. Chem. Chem. Phys. 2015, 17, 9001-9014.

(44) Ekaterina, B. Z.; Oleg, V. B. Theory of steric stabilization of colloid systems by grafted polymers. Makromol. Chem., Macromol. Symp. 1991, 44, 275-284.

(45) Benoit, D. N.; Zhu, H.; Lilierose, M. H.; Verm, R. A.; Ali, N.; Morrison, A. N.; Fortner, J. D.; Avendano, C.; Colvin, V. L. Measuring the Grafting Density of Nanoparticles in Solution by Analytical Ultracentrifugation and Total Organic Carbon Analysis. Anal. Chem. 2012, 84, 9238-9245.

(46) Scheutjens, J. M. H. M.; Fleer, G. J. Statistical theory of the adsorption of interacting chain molecules. 1. Partition function, segment density distribution, and adsorption isotherms. J. Phys. Chem. 1979, 83, 1619-1635.

(47) Fleer, G. J.; Cohen Stuart, M. A.; Scheutjens, J. M. H. M.; Cosgrove, T.; Vincent, B. Polymers at Interfaces; Springer Netherlands, 1998.

(48) Flory, P. J. Principles of Polymer Chemistry; The George Fisher Baker Non-Resident Lectureship in Chemistry at Cornell University; Cornell University Press, 1953.

(49) Fleer, G. J. Polymers at interfaces and in colloidal dispersions. Adv. Colloid Interface Sci. 2010, 159, 99-116.

(50) Leermakers, F. A. M.; Sprakel, J.; Besseling, N. A. M.; Barneveld, P. A. On the curvature dependence of the interfacial tension in a symmetric three-component interface. Phys. Chem. Chem. Phys. 2006, 9, 167-179.

(51) Hilz, E.; Leermakers, F. A. M.; Vermeer, A. W. P. A selfconsistent field study of a hydrocarbon droplet at the air-water interface. Phys. Chem. Chem. Phys. 2012, 14, 4917-4926.

(52) Hill, T. L. Thermodynamics of Small Systems, Parts I \& II; WileyVCH Verlag, 1965; Vol. 3.

(53) Lauw, Y.; Leermakers, F. A. M.; Cohen Stuart, M. A. SelfConsistent-Field Analysis of the Micellization of Carboxy-Modified Poly(ethylene oxide)-poly(propylene oxide)-poly(ethylene oxide) Triblock Copolymers. J. Phys. Chem. B 2006, 110, 465-477.

(54) Schutjens, J. M. H. M.; Fleer, G. J.; Stuart, M. A. C. End effects in polymer adsorption: A tale of tails. Colloids Surf. 1986, 21, 285306.

(55) Feynman, R.; Leighton, R.; Sands, M. The Feynman Lectures on Physics; Addison-Wesley Pub. Co., 1965; Vol. I, Chapter 14. http:// www.feynmanlectures.caltech.edu/I_14.html.

(56) Helfand, E.; Tagami, Y. Theory of the interface between immiscible polymers. J. Polym. Sci., Part B: Polym. Lett. 1971, 9, 741746. 